\title{
Energodynamic theory of gravitation
}

\begin{abstract}
The article substantiates the new concept of gravity and levitation, emanating from the existence in the Universe of the forces of both repulsion and repulsion, depending on the sign of the density gradient of the space environment. According to this concept, gravitational energy has both potential (gravistatic) and kinetic (gravidynamic) components. The forces corresponding to them are proportional to the gradients of density and the oscillatory velocity of the medium, creating gravity. In some cases, these forces can be mutually compensated, causing weightlessness and levitation. Consequences of the new theory of gravity include the Newtonian law of gravity, the prediction of the existence of "strong" gravity and gravitational equilibrium, the possibility of utilizing gravitational energy and the formation of all forms of celestial bodies due to this energy.
\end{abstract}

Keywords: nature of gravity; Newton's law; busting and pushing away; gravitational balance and stability; strong gravity, free energy of gravity.
Volume 3 Issue I - 2019

VA Etkin
Integrative Research Institute, Israel

Correspondence: VA Etkin, Integrative Research Institute, Gtula,39;3339605, Haifa, Israel, Tel (972)547595285, Email v_a_etkin@bezeqint.net

Received: February 21, 2019 | Published: March 29, 2019

\section{Introduction}

The causes of nature and the nature of gravity interested humanity from ancient times. Historically, the first of extant concepts of gravity was the vortex model of Democritus. ${ }^{1}$ He considered gravity to be an "emergent" property arising from the appearance of vortices in a medium, later called ether. Aristotle also adhered to the same concept, who explained the many observable phenomena by the appearance of whirlwinds in this medium.

With a new acuity, the question of the origin of gravity arose after I. Newton, on the basis of the laws of the motion of the planets found by Kepler's, derived his famous law of world wideness. ${ }^{2}$ The reason is that in this law, the force of force has acted as an innate property of the "gravity" of bodies, eternal inherent in any bodies, just like mass and inertia. Therefore, many thinkers of the 18th century, including Descartes, Huygens, and Kelvin, remained, in spite of Newton, supporters of the vortex concept of gravity.

Meanwhile, back in 1690, the Geneva mathematician N. Fatio proposed a simple "kinetic" theory of gravity, which gave a different explanation to the formula of Newton's power. He suggested that the universe is filled with the smallest corpuscles that move at very high speeds randomly and straightforwardly in all directions, and showed that the flux density of these particles decreases in proportion to the square of the distance. His ideas, which became well-known by correspondence with scientists of the time, were not in vain, and in 1756 Le Sage $^{3}$ published the "pushing" concept of gravity based on his ideas. His theory gave a mechanical explanation to a number of phenomena, and in the context of the just-discovered kinetic theory of gases V. Thomson, 1873 became a subject of heightened interest. However, critics of Le Sage's theory found many weak points in it. In addition to logical difficulties, it was noted that moving bodies would have to undergo noticeable inhibition by flying corpuscles, which in fact was not observed. Later, D. Maxwell (1865) and A. Poincaré (1908) showed by calculations that in the Le Sage model, the energy of these particles will certainly turn into heat and not only melt anybody, but also incinerate all the planets. Not consistent with astronomical observations and the absence of changes in the trajectory of the Earth and the Moon, caused by their screening effect during lunar and solar eclipses. As a result, by the beginning of the nineteenth century, the Le Sage model was declared untenable. ${ }^{4}$

Numerous attempts to present gravity as a manifestation of the action of electromagnetic forces could not stand the test of time, although ideas of this kind were expressed several times in the XIX century (M. Faraday, J. Maxwell, X. Lorenz, O. Heviside, etc.). Proponents of this concept came from the similarity of the laws of Newton and Coulomb. However, gravitational forces act between any bodies, while electric - only between charged ones. Secondly, gravitational forces are incomparably smaller than electric ones and manifest themselves mainly in the presence of objects of astronomical scales. Third, in gravity, only the forces of attraction are known, whereas in electricity there are repulsive forces. Fourth, electric forces depend on the speeds of bodies (magnetic interaction), and in gravity such effects are unknown. Finally, the electric field is shielded by conductors, whereas gravitational screens do not exist. To explain these differences could not one "electromagnetic" theory of gravity.

The search for the nature of gravity continued in the twentieth century. The most successful theory of gravity is considered to be GR of A. Einstein..$^{5}$ It postulates that gravitational and inertial forces are of the same nature. Therefore, A. Einstein suggested that gravity is due to other reasons, namely, the deformation of the space-time itself. In this regard, the concept of force became superfluous, and the curvature of space became the cause of the motion of any bodies. As in the "Principles" of I. Newton, in GRT nothing is said about the mechanism of gravitational interactions. Therefore, Einstein's attempt to combine gravity with electromagnetism in the framework of GR, even if successful, could hardly figure out the physical nature of such "electrogravity" forces.

The formalism of general relativity gave rise to many even less "physical" theories of gravity. These include quantum, nonmetric, vector, scalar-tensor, multidimensional, string, etc. theory. ${ }^{6}$ In them, the gravitational interaction is usually attributed to its own field and its own particle - the carrier of this field. However, if for other fundamental interactions their material carriers (mesons, bosons, and photons) are discovered and studied experimentally, then no carriers of gravity have yet been found. 
In this connection, it is of interest to develop a different, energodynamic concept of gravity, based on the latest astrophysical data, which confirmed the prevalence in the Universe of the antipode of ordinary "luminous" (baryonic) matter. ${ }^{7}$ The existence of such an environment was somehow recognized by all previous theories, calling it at different times "ether", "hidden mass", "physical vacuum", "field", "dark matter", "dark energy", etc. Various models endowed this environment with a number of contradictory hypothetical properties. In contrast, the proposed energodynamic concept relies only on the pervasive character of this substance recognized by all theories (including the celestial bodies derived from it) and on the experimental fact of its participation in the gravitational interaction. All other properties of this matter, which we will call for brevity "unstructured" (non-barion), are subject to justification in the framework of the proposed concept. Its goal is to show from the standpoint of energy dynamics [8] that gravity is by no means a congenital property of material bodies, but arises due to the heterogeneity of the Universe, and to obtain on this basis a number of non-trivial consequences of general physical value.

\section{Methodological features of energodynamics applied to the universe}

Energodynamics as a unified theory of the processes of transfer and transformation of any form of energy as opposed to quantum mechanics and general relativity develops, rather than replaces, the Newtonian concept of force. Its research method is based, like classical thermodynamics, ${ }^{7}$ on the properties of the total energy differential as the most general function of the system state. Like it, energy dynamics operates with the parameters of the systems as a whole, without breaking the continuum like the theory of irreversible processes (TIP) into an infinite number of conditionally equilibrium elements. ${ }^{8}$ This allows it to preserve the deductive (systemic) approach to research (from the general to the particular and from the whole to the part), which is characteristic of classical thermodynamics. It becomes especially relevant in connection with the discovered loss of "system-forming" properties when the system is divided into an infinite number of conditionally equilibrium elements1). This is achieved by introducing the missing parameters of the spatial heterogeneity of the studied systems as a whole. Such an approach requires consideration of the Universe as a system that includes "everything that exists," that is, the entire set of interacting (mutually moving) material objects. Such a system, by definition, is closed (does not exchange matter with the environment), is closed (not subject to external forces F), and isolated (does not exchange energy with the external environment). Nevertheless, the processes in the universe have not ceased for at least 14 billion years. This means that it develops, bypassing the state of equilibrium. In this case, the basic postulate of classical thermodynamics, according to which the isolated system reaches an equilibrium state, in which all macroprocesses are terminated, for the final, ${ }^{7}$ is not applicable to the Universe as a whole. Energy dynamics can not be based on the postulates of a local equilibrium consumer goods, according to which the volume elements of a nonequilibrium continuum are described by the same set of variables as in equilibrium. ${ }^{8}$ The latter is due to the fact that the processes of interest occur in these "elements" of the volume.

Further, the concepts of heat transfer and external work, external kinetic $E^{k}$ and potential energy $E^{p}$, are not applicable to the Universe as an isolated system, and its internal energy U from the "dispersed" (out of working) part of the total energy ${ }^{7}$ itself becomes full energy. In this case, all its forms become components of the internal energy, and the 1st principle of thermodynamics, expressing the law of its conservation through the heat $Q$ and the work $W$, becomes unacceptable. At the same time, energodynamics, in contrast to TIP, cannot exclude from consideration a reversible component of real processes. All this required the construction of energy dynamics on its own conceptual basis, decisively breaking with the assumption that the local equilibrium is maintained under the conditions of real (nonstatic) processes.

The main methodological feature of energy dynamics is the rejection of idealization of processes, as well as of hypotheses and postulates in the foundations of the theory. Therefore, when describing the properties of an object of study, energodynamics does not adhere a priori to any model of the Universe and the matter filling it, dividing the latter, if necessary, only into a structured (baryon) and unstructured (non-barion) component. In this case, the first is understood as ordinary (observable) matter, and under the second, the predominant part of it, which participates only in the gravitational interaction and therefore remains invisible. ${ }^{10}$

An important role in building energodynamics on such principles belongs to the axiom of distinguishability of processes, which asserts the possibility of distinguishing independent objects in the object of study by those special, phenomenologically distinct and irreducible to other state changes that they cause. This axiom allows one to prove "by contradiction" a theorem, according to which the number of arguments for the energy $U$ of a nonequilibrium system is equal to the number of independent processes occurring in it. Among them, a special place is occupied by the class of nonequilibrium redistribution of extensive parameters $\Theta_{i}$ first introduced by energy dynamics (mass $M$, numbers of moles of $k$-th substances or phases $N_{k}$, entropy $S$, charge $\Theta_{\mathrm{e}}$, momentum $\mathbf{P}$, its moment $\mathbf{L}$, etc.) over system $V$. A distinctive feature of such processes is their opposite direction in various parts (regions, phases, components) of the system, which makes them non-equilibrium even during quasistatic (infinitely slow) flow. The inevitability of such processes can be verified by selecting in a non-uniform object of study a subsystem with volumes $V^{\prime}$ and $V^{\prime \prime}$, within which the density $\rho_{\mathrm{i}}=\mathrm{d} \Theta_{\mathrm{i}} / \mathrm{dV}$ of any extensive parameter of the system $\Theta_{\mathrm{i}}$ is greater than or less than their average value $\overline{\rho_{\mathrm{i}}}=\mathrm{V}^{-1} \int \rho_{\mathrm{i}} \mathrm{dV}=\Theta_{\mathrm{i}} / \mathrm{V}$. Then, by virtue of the obvious equality $\Theta_{\mathrm{i}}=\int \rho_{\mathrm{i}} \mathrm{dV}=\int \rho_{\mathrm{i}}{ }^{\prime} \mathrm{d} \mathrm{V}^{\prime}+\int \rho_{\mathrm{i}}{ }^{\prime \prime} \mathrm{V}^{\prime \prime}=\int \overline{\rho_{\mathrm{i}}} \mathrm{dV}$, we have:

$$
\int\left(\rho_{\mathrm{i}}{ }^{\prime}-\overline{\rho_{\mathrm{i}}}\right) d \mathrm{~V}^{\prime}+\int\left(\rho_{\mathrm{i}}{ }^{\prime}-\overline{\rho_{\mathrm{i}}}\right) \mathrm{dV} \mathrm{V}^{\prime \prime}=0
$$

It follows that in a non-uniform system there are always subsystems (regions, phases, components) in which this deviation $\rho_{\mathrm{i}}{ }^{\prime}$ and $\rho_{\mathrm{i}}$ " has the opposite sign. This provision, called in ${ }^{9}$ the principle of opposing processes, expresses the essence of the dialectical the law of "unity and struggle of opposites." It excludes the possibility of investigating such processes by algebraically summing changes in individual parts of the system, which makes the systems approach not only desirable but necessary.

The flow in inhomogeneous environments of the processes of redistribution requires finding their coordinates, i.e., parameters, the change of which is a necessary and sufficient sign of their flow. To find them, we consider the general case of redistribution of the density $\rho_{\mathrm{i}}(\mathbf{r}, \mathrm{t})=\partial \Theta_{\mathrm{i}} / \partial \mathrm{V}$ by any extensive parameter $\Theta_{\mathrm{i}}$ (Figure 1). This distribution corresponds to the density curves $\rho_{\mathrm{i}}(\mathbf{r}, \mathrm{t})$ and potential $\psi_{i}$, which, for clarity, are represented as a function of the radius vector of a point of the field $\mathbf{r}$. As follows from the figure, if 
the distribution of $\Theta_{i}$ as a quantitative measure of the carrier of the $i$-th form of energy from uniform with a density deviates, some of its quantity $\Theta_{i} *$ is transferred from one part of the system to another in the direction indicated by the dotted arrow. Such a "redistribution" of the extensive value $\Theta_{i}$ causes a shift of its center from the initial position $\mathbf{R}_{\hat{\mathbf{i}}}=\Theta_{1}^{-1} \int_{\mathrm{Q}}^{\overline{\mathbf{r}} \mathrm{dV}}$ to the current $\mathbf{R}_{\mathrm{i}}=\Theta_{\mathrm{i}}^{-1} \int \rho_{\mathrm{i}} \mathbf{r d V}$ and the occurrence of some "distribution moment" $\mathbf{Z}_{i}$ :

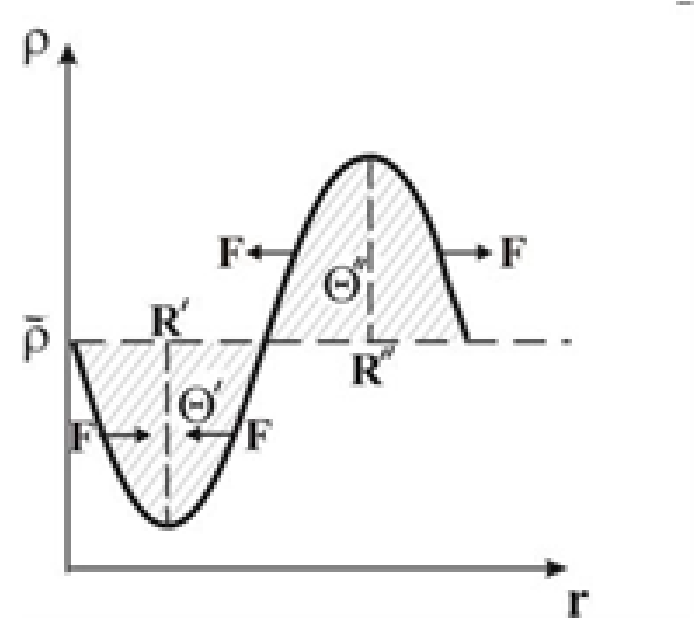

Figure I Wave formation in non-barion matter.

$$
\mathbf{Z}_{i}=\Theta \Delta \mathbf{R}_{i}=\int_{V}\left[\tilde{\mathrm{n}}_{i}(\mathbf{r}, t)-\tilde{\mathrm{n}}_{i}(t)\right] \mathbf{r} d V
$$

Thus, the process of redistribution of any energy carrier $\Theta_{i}$ is characterized by its center displacement vector $\Delta \mathbf{R}_{i}=\mathbf{R}_{i}-\mathbf{R}_{i}$. This means that the state of a nonequilibrium system is characterized by twice the number of state variables: $\mathrm{U}=\Sigma_{\mathrm{i}} \mathrm{U}_{\mathrm{i}}\left(\Theta_{\mathrm{i}}, \Delta \mathbf{R}\right) .{ }^{1}$ In this case, its total differential can be represented as an identity: ${ }^{9}$

$$
\mathrm{dU} \equiv \Sigma_{\mathrm{i}} \Psi_{\mathrm{i}} \mathrm{d} \Theta_{\mathrm{i}}-\Sigma_{\mathrm{i}} \mathbf{F}_{\mathrm{i}} \cdot \mathrm{d} \mathbf{R}_{\mathrm{i}},
$$

where $\Psi_{i} \equiv\left(\partial \mathrm{U} / \partial \Theta_{i}\right)$ is the averaged value of the generalized potential (absolute temperature $T$ and pressure $p$, chemical $\mu_{\mathrm{k}}$, electric $\varphi$, gravitational $\psi_{\mathrm{g}}$, and other potentials $) ; \mathbf{F}_{\mathrm{i}} \equiv-\left(\partial \mathrm{U} / \partial \mathbf{R}_{\mathrm{i}}\right)$ - forces in their general physical understanding as a gradient of the $i$-th form of energy, taken with the opposite sign. All such forces for isolated systems are internal, so their specific values $\mathbf{F}_{\mathrm{i}} / \Theta_{\mathrm{i}}$ should be called strengths and denoted by $\mathbf{X}_{i}$. Under the conditions $\Theta_{\mathrm{i}}=$ const, this corresponds to the expression $\mathbf{X}_{\mathrm{i}}=\Theta_{\mathrm{i}}^{-1}\left(\partial \mathrm{U} / \partial \mathbf{R}_{\mathrm{i}}\right)=-\left(\partial \mathrm{U} / \partial \mathbf{Z}_{\mathrm{i}}\right)$.

A further application of energy dynamics is the use of identity (3) along with the uniqueness conditions (the conservation laws for parameters $\Theta_{\mathrm{i}}$, the equations of state $\Psi_{\mathrm{i}}=\Psi_{\mathrm{i}}\left(\Theta_{\mathrm{i}}, \mathbf{R}_{\mathrm{i}}\right)$ and transfer $\dot{\mathbf{Z}}_{\mathrm{i}}=\dot{\mathbf{Z}}_{\mathrm{i}}\left(\Theta_{\mathrm{i}}, \mathbf{R}_{\mathrm{i}}\right)$, initial and boundary conditions). In the conditions of uniqueness, any hypotheses, postulates, and models are allowed, since they are subject to experimental verification. The uniqueness of such a method consists not only in the fact that all fundamental disciplines (including nonequilibrium thermodynamics) are its particular cases ${ }^{11}$ but also in the fact that the consequences obtained prior to the application of the uniqueness conditions have the property of immutable truths. Consider some of them.

${ }^{1}$ In a more general case, when a change in the direction of the displacement vector is taken into account, as well as the vibrational form of the energy, the number of degrees of freedom can become quadrupled.

\section{Gravity as an emergent property}

Everything objectively existing in this world is called matter. The centuries-old experience of studying its properties indicates that matter is in motion all the time. As long as it has a single property the density $\rho$ as a function of spatial coordinates (radius vector $r$ ), this movement is manifested in the fluctuation $\rho(\mathrm{r})$ about its mean value $(t)$. When matter is distributed evenly, this oscillation is characterized by the same deviation of $\rho(r)$ from $(t)$ in both directions, i.e. It has nature of the harmonic wave (Figure 1).

In such a wave, a reciprocal - forward displacement of the energy carrier $\Theta^{\prime}$ (in this case, the mass $M$ ) in both directions from the equilibrium state to a distance of $\left|\Delta \mathbf{R}_{v}\right|=\lambda / 2$. It is carried out for the period of oscillation, the opposite of its frequency $v$. In this case, the average speed of the oscillatory movement of the mass of the wave $M$ in this process $\bar{v}_{v}=\lambda v$, which then determines the speed of its propagation in space, called the speed of light, $c$. In contrast to the speed of a traveling wave, the oscillatory velocity $v_{v}$ changes from zero in the antinodes of the wave to a maximum at its nodes, and in the general case is a function of the density of the medium $v_{v}=v_{v}(\rho)$.

The density of the vibrational energy $\rho^{k}$, is in this case, the value $\rho^{k}=\rho \tilde{n}^{2} / 2$. Since in the zone of the antinode of the wave the kinetic energy $\rho^{k}$ is completely converted into potential $\rho^{\mathrm{p}}$, their sum remains unchanged and equal to $\rho \tilde{n}^{2}$.

If we take the offset module $\left|\Delta \mathbf{R}_{v}\right|$ for the amplitude of the longitudinal wave $\mathrm{A}_{v}$, we come to the well-known expression for the wave energy density: ${ }^{11}$

$$
\rho^{\mathrm{k}}=\rho \ddot{\mathrm{A}} \ddot{z}^{2} \mathrm{i} \mathrm{v}^{2} / 2,\left({ }^{-3}\right),
$$

Thus, the total energy of the primary matter $U=M c^{2}$ includes in the general case the kinetic component $U^{k}$, which it is expedient to call the gravidynamic component, and the potential component $U^{p}$, which we will call the gravistatic one by analogy. Before A. Einstein, the expression $U=M c^{2}$ was referred to the ether as the "primary" form of matter, from which all other forms of matter were formed (N. Umov, 1873; J. Thomson, 1881; O. Haviside, 1990; A. Poincare, 1900; F. Hasenorl, 1904 [1]). A. Einstein (1905) extended this expression to all forms of energy and matter and, putting $c=$ const, began to treat the proportionality of mass and energy as the principle of their equivalence.

We now apply identity (3) to any region of the Universe with a constant volume $V$, whose mass $\mathrm{M}=\int \rho \mathrm{dV}$ increases in the process of matter flowing into it from the outside. Expressing the energy of the region $\mathrm{U}=\rho_{\mathrm{u}} \mathrm{V}$ through $\rho_{\mathrm{u}}$ and using the expression known from wave theory for the propagation speed of oscillations in an elastic medium as a function of its density $v_{v}{ }^{2}(\rho)=\left(\partial \rho_{u} / \partial \rho\right),{ }^{11}$ from identity (3) as $\bar{v}_{v}=\tilde{n}$ we find the local gravitational potential of this region:

$$
\Psi_{\mathrm{g}} \equiv(\partial \grave{\mathbf{U}} / \partial \quad)_{\mathrm{V}}=\left(\partial \rho_{\mathrm{u}} / \partial \rho\right)=\tilde{\mathrm{n}}^{2}
$$

From identity (3) it is easy to find the strength of the gravitational field $\mathbf{X}_{\mathrm{g}}=-\mathbf{g}$, if we take into account that for the wave $\mathrm{d} \mathbf{Z}_{\mathrm{v}}=\mathbf{M d} \mathbf{R}_{v}=\rho \mathrm{Vd} \mathbf{r}::^{12}$

$$
\mathbf{X}_{\mathrm{g}}=-\tilde{\mathrm{n}}\left(\partial \mathrm{U} / / \partial \mathbf{Z}_{\mathrm{m}} \mathrm{i}\right) \tilde{\mathrm{n}}=-{ }^{2} \nabla \rho \rho\left({ }^{-2}\right)
$$

This expression represents the energodynamic form of the law of gravity. According to it, the gravitational field arises due to the 
inhomogeneous distribution of the density of matter in space, that is, it is an emergent property. At the same time, the gravitational force $\mathbf{X}$ is always directed against the density gradient of matter потомур and therefore can have a different size and sign in different regions of the Universe depending on the magnitude and sign of the density gradient of matter in this region of space..$^{12}$ In other words, gravitational forces can be both attractive and repulsive forces, depending on the nature of the mass distribution in space.

This circumstance in no way follows from the law of Newton, who considered gravity in the scalar approximation and considered the interaction force $F_{g}=G m M / R^{2}$ independent of their mutual orientation in space. In this case, the gravitational potential $\psi_{\mathrm{g}}=\psi_{\mathrm{g}}(\mathrm{R})$, i.e. is determined only by the distance $R$ to the center of the "fieldforming" mass $M$. Nevertheless, expression (6) does not contradict it, if this potential $\psi_{\mathrm{g}}$ is also expressed as a function of density $\rho$ and considered it, like èg, positive. To do this, replace the "test" mass $\mathrm{m}$ with a "test" (unit) volume $V$ with radius $R$, for which this potential is the same at any point on its surface and is equal to. However, the expression (6) does not contradict him, if this potential $\psi_{\mathrm{g}}$ also expressed as a function of density $\rho$ and consider it, like $\Psi_{\mathrm{g}}$, positive. To do this, replace the "test" mass $m$ with a "test" (unit) volume $V_{c}$ with radius $R_{c}$, for which this potential is the same at any point on its surface and is equal to

$$
\psi_{\tilde{\mathrm{g}}}=(\mathrm{GV} / \mathrm{R}) \rho .
$$

Hence, taking into account the constancy of the expression in brackets, it follows that the acceleration $\mathbf{g}=-\mathbf{X}_{g}$ can be expressed as a function of the density gradient of the medium

$$
\mathbf{g}=\left(\mathrm{G}_{\mathrm{N}} \mathrm{V}_{\tilde{\mathrm{n}}} / \mathrm{R}_{\tilde{\mathrm{n}}}\right) \nabla \rho / \rho=\psi_{\mathrm{g}} \nabla \rho / \rho,
$$

Thus, Newton's law in a continuous medium can also be expressed in terms of a density gradient by the same relation (6), replacing only the proportionality coefficient $\Psi_{\mathrm{g}}$ by $\psi_{\mathrm{g}}$. However, now this law has acquired a vector form and has become a consequence of the law of gravity (6) for continuous media in the particular case of pair wise interaction of bodies in empty space. According to this law, with an increase in the density of the medium $\rho$, all other conditions being equal, the forces $\mathbf{X}_{g}$ weaken, which contradicts the general theory of relativity with increasing curvature with increasing mass. Consequently, the statement of GR, which is the cause of the curvature of space, does not correspond to Newton's law in the form of (8). At the same time, the laws of gravity in the form of (6) and (8), as well as the energy dynamic identity (3), emphasize that gravity is not the "innate" property of the substance filling the space. This confirms the correctness of R. Feynman, who considered that the force field is not a physical reality, but a mathematical function introduced for its characteristics, ${ }^{13}$ as well as E. Verlinde, ${ }^{14}$ protesting against the interpretation of gravity as an inherent property. From the standpoint of energy dynamics, gravity is a consequence of the inhomogeneous distribution of matter in the Universe, caused by the oscillatory nature of its motion. The large-scale gravity waves recently discovered by a number of scientific collaborations ${ }^{15}$ and the so-called baryon acoustic oscillations of the primary plasma of the Universe ${ }^{16}$ indicate the wave nature of this process.

\section{Consequences of the energodynamic theory of gravity}

The heuristic value of the generalized law of gravity consists primarily in predicting the existence of gravitational equilibrium, characterized by the absence of the resulting force $\mathbf{F}_{g}$. As follows from laws (6) and (8), the condition for the onset of gravitational equilibrium, i.e. zero gravity $\mathbf{F}_{g}=M \mathbf{g}=0$, is the vanishing of the density gradient of the matter:

$\nabla \rho=0$

regardless of the value of the density itself $\rho$.

This position also did not follow from the law of Newton, according to which the forces $\mathbf{F}_{g}$ reach zero only at an infinite distance from the field-forming bodies. Meanwhile, the presence of such an equilibrium was evidenced by the phenomenon of libration, when a small celestial body could stay for a long time in the space between the masses without changing its position.

Moreover, recent astronomical studies conducted in the framework of the "large" Sloanovsky digital sky survey (SDSS) have found vast voids in space ("voids"), the density of galaxies and their clusters in which is an order of magnitude lower than their average density. ${ }^{17}$ This indicates the absence in them of conditions for the formation of stars and their clusters, which according to (8) is the presence of a gradient of their density $\rho$. Another non-trivial consequence of the energodynamic theory of gravity is the discovery that gravitational forces are hardly inferior in their intensity to intranuclear forces. You can make sure that the gravistatic forces are many orders of magnitude greater than the Newtonian forces of force by comparing the potential $\Psi_{\mathrm{g}}=\tilde{\mathrm{n}}^{2} \approx 9.10^{16} \mathrm{~J} / \mathrm{kg}$ with the Newtonian potential $\mathrm{g}$, for example, on the surface of the Sun where it is maximum and equal to $\psi_{\mathrm{g}}=1,906.10^{11} \mathrm{~J} / \mathrm{kg} .{ }^{18}$ This gives additional arguments in favor of the existence of "strong" gravity. ${ }^{19}$

The next non-trivial consequence is the discovery of gravitational component $\mathrm{U}_{\mathrm{g}}{ }^{\mathrm{v}}$ in gravitational energy, which corresponds to the concept of free energy. This component can perform work without any movement of celestial bodies, which eliminates the violation of the law of conservation of energy by the so-called "superunit" devices that use gravitational energy. ${ }^{20}$

This allows you to take a fresh look at the emergence of "excess heat generation" when operating oxygen - hydrogen electrolysers on ordinary and heavy water (at polarization reversal of nonlinear dielectrics and magnetics; in vortex heat generators; in the recombination of hydrogen; during plasma and plasma-chemical dialysis, with "sonoluminescence", etc. Thus, it also becomes possible to explain the "production" of thermal energy in these facilities by "cold nuclear fusion", not by extracting energy from the physical vacuum as the lowest energy state, but in a more prosaic way.

The existence of gravitational equilibrium explains the anomalous slowing down of the movement of space probes of the Pioneer and Voyager 1 and 2 types in a natural way as a result of their entry into a more uniform area of space. The presence of repulsive forces in outer space makes it possible to abandon the attraction of exotic "dark energy".

Finally, the invariance of the sign of the gravitational forces $\mathbf{F}_{g}$ with a spontaneous imbalance of local equilibrium explains the steady compression of celestial bodies until the appearance of a singularity in them. This allows us to explain the flashes of "supernovae" and phenomena like the "Big Bang" not towards the Universe as a whole, but towards its visible part, without rejecting its ability to develop, bypassing the equilibrium state. ${ }^{21}$ 


\section{Acknowledgments}

None.

\section{Conflicts of interest}

Author declares that there is no conflict of interest.

\section{References}

1. Whittaker E. A History of the Theories of Aether and Electricity. The Modern Theories 1900-1926. London: Thomos Nelson; 1953.

2. Newton I. Mathematical Principles of Natural Philosophy. Petrograd; 1916.

3. Le Sage GL. Letter à une académicien de Dijon. Mercure de France; 1756. $153-171 \mathrm{p}$.

4. Roseveare NT. Mercury's perihelion. From Le Verrier to Einstein. Clarendon Press Oxford 1982 Einstein A. Die Grundlage der allgemeinen Relativitätstheorie. Annalen der Physik. 1916;354(7):769-822.

5. Misner C, Torn K, Wheeler J. Gravity. Volume 3: Mir; 1977.

6. Ade PAR, Aghanim N, Alves MIR, et al. Planck 2013 results. I. Overview of products and scientific results. Astrophysics. 2014;1303:5062

7. Gyarmati I. Non-Equilibrium Thermodynamics. Field Theory and Variation Principles. Berlin: Springer Verlag; 1970.184 p.

8. Etkin VA. Synthesis of the fundamentals of engineering disciplines (Energodiamics approach to the integration of knowledge). Lambert Academic Publishing; 2011. 290 p

9. Bazarov IP. Thermodynamics. New York: Pergamon Press; 1964.
10. Etkin V. Energodynamics (Thermodynamic Fundamentals of Synergetics). New York; 2011, 480 p.

11. Etkin V. Gravitational repulsive forces and evolution of universe. Journal of Applied Physics. 2016;8(4):43-49.

12. Feynman R, Leighton R, Sands M. The Feynman Lectures on Physics. T. 6. M .: Mir; 1966.

13. Verlinde EP. On the Origin of Gravity and the Laws of newton. Journal of High Energy Physics. 2011;4(29):1-10.

14. Erik P Verlinde. On the Origin of Gravity and the Laws of Newton. High Energy Physics-Theory. 2011.

15. http://ria.ru/science/20160211/1372881614.html

16. Eisenstein DJ, Zehavi I, Hogg DW, et al. Detection of the Baryon Acoustic Peak in the Large-Scale Correlation Function of SDSS Luminous Red Galaxies. Astrophysics; 2005.

17. BOSS: Dark Energy and the Geometry of Space. USA: SDSS III.

18. Etkin VA. Generalized Law of Gravitation. World Scientific News. 2017;74:272-279.

19. Salam A. Strong Interactions, Gravitation, and Cosmology. Cambridge: MIT Press; 1974.

20. Etkin VA. Theoretical basis of fuel-free energy. Saarbrüken (Canada): Altaspera Publications; 2013.

21. Etkin VA. New Criteria of Evolution and Involution of the Isolated Systems. International Journal of Thermodynamics. 2018;21(2):120-126.

22. Crawford F. Waves. Berkeley Physics course Vol 3. NewYork: McGraw-Hill; 1968. 600 p. 